\title{
Prognostic Value of Myocardial Damage Markers in Patients with Chronic Heart Failure with Atrial Fibrillation
}

\author{
Yoichiro Otaki, Takanori Arimoto, Hiroki Takahashi, Shinpei Kadowaki, Daisuke Ishigaki, \\ Taro Narumi, Yuki Honda, Tadateru Iwayama, Satoshi Nishiyama, Tetsuro Shishido, \\ Takehiko Miyashita, Takuya Miyamoto, Tetsu Watanabe and Isao Kubota
}

\begin{abstract}
Objective The aim of the present study was to examine the relationship between myocardial damage caused by atrial fibrillation (AF) and subsequent cardiovascular events in AF patients with chronic heart failure (CHF).

Methods and Results We measured the serum levels of heart-type fatty acid binding protein (H-FABP) and high-sensitivity troponin $\mathrm{T}$ in 402 consecutive $\mathrm{CHF}$ patients with chronic $\mathrm{AF}$ (CHF-AF, $\mathrm{n}=201$ ) or sinus rhythm (CHF-SR, n=201). The patients with CHF-AF had higher H-FABP and troponin T levels than those with CHF-SR. In order to examine the prognostic value of myocardial damage markers in CHF-AF and CHF-SR patients, we followed the above patients and analyzed their clinical outcomes. A multivariate Cox proportional hazard analysis revealed that both the serum H-FABP and troponin $\mathrm{T}$ levels independently predicted subsequent cardiovascular events. A Kaplan-Meier analysis demonstrated that the rate of cardiovascular events was higher in the patients with elevated H-FABP and troponin T levels. The optimal cut-off values for the myocardial damage markers of cardiovascular events were higher in the CHF-AF patients than in the CHF-SR patients (H-FABP, 5.4 vs. $4.6 \mathrm{ng} / \mathrm{mL}$ and troponin T, 0.030 vs. $0.013 \mathrm{ng} / \mathrm{mL}$ ).

Conclusion Myocardial damage advances in association with the presence of $\mathrm{AF}$ and is associated with subsequent cardiovascular events in both CHF-AF and CHF-SR patients. In this study, the cut-off values for the myocardial damage markers were higher in the CHF-AF patients than in the CHF-SR patients.
\end{abstract}

Key words: atrial fibrillation, heart type fatty acid binding protein, troponin $\mathrm{T}$

(Intern Med 53: 661-668, 2014)

(DOI: 10.2169/internalmedicine.53.1293)

\section{Introduction}

Chronic atrial fibrillation (AF) is a frequently observed tachyarrhythmia that is associated with a poor prognosis in patients with chronic heart failure $(\mathrm{CHF})(1,2)$. Accumulating evidence indicates that AF deteriorates the cardiac function by inducing tachycardia, reduced ventricular filling and atrial contractile dysfunction $(3,4)$. Conversely, CHF has been reported to be a risk factor for the new onset of AF through atrial dilatation, local conduction disturbances, atrial fibrosis and atrial volume overload $(5,6)$. Although the precise mechanisms remain a matter of debate, the interaction between $\mathrm{AF}$ and $\mathrm{CHF}$ is a vicious cycle (7). Furthermore, while it is known that myocardial damage plays a pivotal role in cardiac remodeling $(8,9)$, the impact of AF on myocardial damage remains to be elucidated.

The aim of the present study was to: 1) evaluate whether myocardial damage, as measured according to the levels of heart-type fatty acid binding protein (H-FABP) and troponin $\mathrm{T}$, is associated with AF and 2) determine whether these markers predict subsequent cardiovascular events in patients 
with CHF-AF and CHF-sinus rhythm (SR).

\section{Materials and Methods}

\section{Study population}

Between January 2003 and December 2009, 630 consecutive patients with CHF were admitted to Yamagata University Hospital for the diagnosis, pathophysiological investigation and treatment or therapeutic evaluation of heart failure. CHF was diagnosed based on echocardiographic, radionuclide and cardiac catheterization findings. The clinical diagnosis of CHF was confirmed by at least two cardiologists. Patients with acute coronary syndrome diagnosed within three months prior to admission were excluded. The diagnosis of chronic AF was defined as an AF episode lasting longer than six months with no rhythm control interventions, according to the guidelines for patients with AF (10).

From the above described population, 201 consecutive CHF patients with AF (CHF-AF) and 201 consecutive CHF patients with CHF-SR were prospectively enrolled in this study.

Venous blood sampling was performed after the patient had been stable without acute decompensated heart failure for at least one week. An experienced cardiologist who had no knowledge of the biochemical data performed twodimensional echocardiography on the day of blood sampling. The physicians were blinded to the results of the biochemical analyses, and optimal medical therapy was independently administered based on symptom improvements and physical examination and chest $\mathrm{X}$-ray findings.

Written informed consent was provided by all patients prior to participation in the study, and the study protocol was approved by the institutional Human Investigations Committee.

\section{H-FABP, troponin T and BNP measurements}

The blood samples used to measure the serum H-FABP levels were centrifuged at $2,500 \mathrm{~g}$ for 15 minutes at $4^{\circ} \mathrm{C}$ within 30 minutes of collection, and the serum was stored at $-70^{\circ} \mathrm{C}$ until the analysis. The H-FABP levels were measured using a two-step sandwich enzyme-linked immunosorbent assay (MARKIT-M H-FABP, Dainippon Pharmaceutical Co. Ltd., Tokyo, Japan) (11, 12). The high-sensitivity troponin $\mathrm{T}$ concentrations were measured using a fourth-generation electrochemiluminescence immunoassay on an Elecsys 2010 automatic analyzer (Elecsystroponin-T, Roche Diagnostics, Tokyo, Japan) (13). Blood samples were also obtained to measure the brain natriuretic peptide (BNP) levels. These samples were transferred to chilled tubes containing $4.5 \mathrm{mg}$ of ethylenediaminetetraacetic acid disodium salt and aprotinin $(500 \mathrm{U} / \mathrm{mL})$ and centrifuged at $1,000 \mathrm{~g}$ for 15 minutes at $4{ }^{\circ} \mathrm{C}$. The clarified plasma samples were frozen, stored at $-70^{\circ} \mathrm{C}$ and thawed immediately prior to the assay. The BNP concentrations were measured using a commercially available radioimmunoassay specific for human BNP
(Shiono RIA BNP assay kit, Shionogi Co., Ltd., Tokyo, Japan) $(11,14)$.

\section{Endpoints and follow-up}

The patients with CHF-AF and CHF-SR were prospectively followed for a median period of 643 days (range, 496-1,056 days) and 488 days (range, 350-1,122 days), respectively. The endpoints were all-cause death and cardiovascular events, including cardiovascular death, defined as death from progressive heart failure, myocardial infarction, cardioembolic stroke or sudden cardiac death, and progressive heart failure requiring rehospitalization. Sudden cardiac death was defined as death without definitive premonitory signs or symptoms, as established by the attending physician.

\section{Statistics}

All values are expressed as the mean \pm SD or median. Continuous and categorical variables were compared using the t-test and chi-square test, respectively. A Cox proportional hazard analysis was performed to determine independent predictors for cardiac events, and significant predictors selected in the univariate analysis were entered into the multivariate analysis. Receiver operating characteristics (ROC) curves for H-FABP and troponin $\mathrm{T}$ were constructed to determine the optimal cut-off values for sensitivity and specificity. The area under the curve for cardiovascular events was calculated according to the trapezoidal rule. Cardiac event-free and survival curves were constructed based on the Kaplan-Meier method and compared using the log-rank test. A value of $p<0.05$ was considered to be statistically significant. All statistical analyses were performed using a standard software program (JMP version 8, SAS Institute Inc., Cary, USA).

\section{Results}

\section{Baseline characteristics of the CHF-SR and CHF-AF patients}

The baseline characteristics of the CHF-SR and CHF-AF patients are presented in Table 1. There were 102 and 99 patients with a New York Heart Association (NYHA) functional class II and III status, respectively. Hypertension and diabetes mellitus were identified in 135 (67\%) and 60 (30\%) of the CHF-AF patients, respectively. The etiology of CHFAF was identified to be non-ischemic heart disease in 176 $(88 \%)$ patients and ischemic heart disease in 25 (12\%) patients. The median levels of H-FABP and troponin $\mathrm{T}$ were 4.7 (3.6-8.2) ng/mL and $0.020(0.005-0.050) \mathrm{ng} / \mathrm{mL}$, respectively.

\section{Comparison of the clinical characteristics between the CHF-SR and CHF-AF patients}

The CHF-AF patients had higher H-FABP and troponin T levels than the CHF-SR patients. In addition, the patients 
Table 1. Comparisons of Clinical Characteristics between CHF-AF and CHF-SR Patients

\begin{tabular}{|c|c|c|c|}
\hline Variables & $\begin{array}{c}\text { CHF-SR } \\
n=201\end{array}$ & $\begin{array}{c}\text { CHF-AF } \\
n=201\end{array}$ & $\mathrm{p}$ value \\
\hline Age & $69 \pm 12$ & $71 \pm 12$ & 0.1582 \\
\hline Male/female & $119 / 82$ & $121 / 80$ & 0.8388 \\
\hline NYHA functional class (II/III) & $102 / 99$ & $102 / 99$ & $>0.9999$ \\
\hline Hypertension & $135(67 \%)$ & $135(67 \%)$ & $>0.9999$ \\
\hline Diabetes mellitus & $57(28 \%)$ & $60(30 \%)$ & 0.7419 \\
\hline Etiology & & & 0.5865 \\
\hline Non-ischemic heart disease & $170(85 \%)$ & $176(88 \%)$ & \\
\hline Ischemic heart disease & $31(15 \%)$ & $25(12 \%)$ & \\
\hline Heart rate & $71 \pm 13$ & $71 \pm 14$ & 0.8048 \\
\hline \multicolumn{4}{|l|}{ Blood examination } \\
\hline eGFR $\left(\mathrm{mL} / \mathrm{min} / 1.73 \mathrm{~m}^{2}\right)$ & $65 \pm 19$ & $64 \pm 27$ & 0.5515 \\
\hline $\mathrm{BNP}(\mathrm{pg} / \mathrm{mL})$ & $383(104-980)$ & $390(164-813)$ & 0.5559 \\
\hline H-FABP (ng/mL) & $4.1(2.9-6.5)$ & $4.7(3.6-8.2)$ & 0.0176 \\
\hline Troponin $\mathrm{T}(\mathrm{ng} / \mathrm{mL})$ & $0.005(0.005-0.014)$ & $0.020(0.005-0.050)$ & 0.0445 \\
\hline \multicolumn{4}{|l|}{ Echocardiography } \\
\hline $\mathrm{LAD}(\mathrm{mm})$ & $43 \pm 4$ & $50 \pm 9$ & $<0.0001$ \\
\hline LVEDD (mm) & $56 \pm 10$ & $55 \pm 10$ & 0.2889 \\
\hline LVEF (\%) & $48 \pm 19$ & $47 \pm 17$ & 0.5457 \\
\hline \multicolumn{4}{|l|}{ Medication } \\
\hline ACEIs and/or ARBs & $149(74 \%)$ & $151(75 \%)$ & 0.1355 \\
\hline$\beta$-blockers & $123(61 \%)$ & $133(66 \%)$ & 0.2997 \\
\hline Calcium channel blockers & $40(20 \%)$ & $52(25 \%)$ & 0.2315 \\
\hline Warfarin & $55(27 \%)$ & $160(80 \%)$ & $<0.0001$ \\
\hline Loop diuretics & $116(58 \%)$ & $159(79 \%)$ & $<0.0001$ \\
\hline \multicolumn{4}{|c|}{$\begin{array}{l}\text { Data are expressed as mean } \pm \text { SD, number (percentage), or median (interquartile range). } \\
\text { ACEIs: angiotensin-converting enzyme inhibitors, AF: atrial fibrillation, ARBs: angiotensin II } \\
\text { recepter blockers, BNP: brain natriuretic peptide, CHF: chronic heart failure, eGFR: estimated } \\
\text { glomerular filtration rate, H-FABP: heart-type fatty acid binding protein, LAD: left atrial } \\
\text { dimension, LVEDD: left ventricular end-diastolic dimension, LVEF: left ventricular ejection } \\
\text { fraction, NYHA: New York Heart Association, SR: sinus rhythm }\end{array}$} \\
\hline
\end{tabular}

with CHF-AF displayed larger left atrial dimensions and had taken warfarin and loop diuretics more frequently than those with CHF-SR. On the other hand, there were no significant differences in age, gender, NYHA functional class, prevalence of hypertension and diabetes mellitus, CHF etiology, heart rate, estimated glomerular filtration rate (eGFR), BNP, left ventricular end-diastolic diameter, left ventricular ejection fraction and use of angiotensin-converting enzyme inhibitors, angiotensin II receptor blockers, $\beta$-blockers or calcium channel blockers between the CHF-AF and CHF-SR patients.

\section{Prognostic value of the H-FABP and troponin T lev- els in the CHF-AF patients}

There were 69 cardiovascular events (35\%), including 24 cardiovascular deaths and 45 episodes of rehospitalization for worsening of heart failure during the follow-up period. The cause of cardiovascular death was worsening of heart failure in 20 patients, myocardial infarction in two patients, cardioembolic stroke in one patient and sudden cardiac death in one patient. There were 38 all-cause deaths among the patients with CHF-AF.

To determine the risk factors for predicting cardiovascular events, we performed univariate and multivariate Cox proportional hazard regression analyses (Table 2). According to the univariate analysis, the H-FABP and troponin $\mathrm{T}$ levels were significantly associated with cardiovascular events.
Furthermore, age and the NYHA functional class, eGFR and left atrial dimension were found to be significantly related to cardiovascular events. A multivariate analysis revealed that the H-FABP and troponin T levels were independent predictors for cardiovascular events after adjusting for age and the NYHA functional class, eGFR and left atrial dimension (HFABP: hazard ratio, 1.566; 95\% confidence interval, 1.2402.003; $\mathrm{p}=0.0002$ and troponin $\mathrm{T}$ : hazard ratio, $1.402 ; 95 \%$ confidence interval, 1.128-1.742; $\mathrm{p}=0.0023$ ) (Table 2).

\section{Prognostic value of the H-FABP and troponin T lev- els in the CHF-SR patients}

There were 61 cardiovascular events (30\%), including 21 cardiovascular deaths and 40 episodes of rehospitalization for worsening of heart failure during the follow-up period. The cause of cardiovascular death was worsening of heart failure in 18 patients, myocardial infarction in one patient and sudden cardiac death in two patients. There were 30 allcause deaths among the patients with CHF-SR.

In order to determine the risk factors for predicting cardiovascular events, we performed univariate and multivariate Cox proportional hazard regression analyses (Table 3). According to the univariate analysis, the level of H-FABP, but not troponin $\mathrm{T}$, was significantly associated with cardiovascular events. Furthermore, age, NYHA functional class, eGFR and BNP were found to be significantly related to cardiovascular events. A multivariate analysis revealed that 
Table 2. Univariate and Multivariate Cox Proportional Hazard Analyses of Predicting Cardiovascular Events in CHF-AF Patients

\begin{tabular}{|c|c|c|c|}
\hline Variables & Hazard Ratio & $95 \%$ confidence Interval & $\mathrm{p}$ value \\
\hline \multicolumn{4}{|l|}{ Univariate analysis } \\
\hline Age (Per 1-year increase) & 1.034 & $1.009-1.059$ & 0.0067 \\
\hline Gender (female vs. male) & 1.143 & $0.708-1.845$ & 0.5848 \\
\hline NYHA functional class (III vs. II) & 2.119 & $1.292-3.484$ & 0.0030 \\
\hline Hypertension & 1.325 & $0.800-2.192$ & 0.2742 \\
\hline Diabetes mellitus & 0.667 & $0.408-1.090$ & 0.1057 \\
\hline Heart rate & 1.073 & $0.832-1.375$ & 0.5954 \\
\hline eGFR (Per 1-SD increase) & 0.649 & $0.464-0.921$ & 0.0134 \\
\hline BNP (Per 1-SD increase) & 1.170 & $0.926-1.469$ & 0.1324 \\
\hline H-FABP (Per 1-SD increase) & 1.799 & $1.515-2.138$ & $<0.0001$ \\
\hline Troponin T (Per 1-SD increase) & 1.421 & $1.218-1.658$ & $<0.0001$ \\
\hline LAD (Per 1-SD increase) & 1.513 & $1.195-1.933$ & 0.0007 \\
\hline LVEDD (Per 1-SD increase) & 1.010 & $0.784-1.318$ & 0.9142 \\
\hline LVEF (Per 1-SD increase) & 1.017 & $0.773-1.267$ & 0.9148 \\
\hline \multicolumn{4}{|l|}{ Multivariate analysis for $H-F A B P$} \\
\hline Age (Per 1-year increase) & 1.010 & $0.984-1.037$ & 0.4422 \\
\hline NYHA functional class (III vs. II) & 1.540 & $0.852-2.786$ & 0.1532 \\
\hline eGFR (Per 1-SD increase) & 0.898 & $0.629-1.274$ & 0.5710 \\
\hline H-FABP (Per 1-SD increase) & 1.566 & $1.240-2.003$ & 0.0002 \\
\hline LAD (Per 1-SD increase) & 1.346 & $1.055-1.718$ & 0.0157 \\
\hline \multicolumn{4}{|l|}{ Multivariate analysis for Troponin $T$} \\
\hline Age (Per 1-year increase) & 1.009 & $0.980-1.039$ & 0.5519 \\
\hline NYHA functional class (III vs. II) & 1.629 & $0.831-3.185$ & 0.1556 \\
\hline eGFR (Per 1-SD increase) & 0.583 & $0.372-0.923$ & 0.0210 \\
\hline Troponin T (Per 1-SD increase) & 1.402 & $1.128-1.742$ & 0.0023 \\
\hline LAD (Per 1-SD increase) & 1.408 & $1.084-1.808$ & 0.0096 \\
\hline
\end{tabular}

Table 3. Univariate and Multivariate Cox Proportional Hazard Analyses of Predicting Cardiovascular Events in CHF-SR Patients

\begin{tabular}{|c|c|c|c|}
\hline Variables & Hazard Ratio & $95 \%$ confidence Interval & $\mathrm{p}$ value \\
\hline \multicolumn{4}{|l|}{ Univariate analysis } \\
\hline Age (Per 1-year increase) & 1.041 & $1.014-1.069$ & 0.0024 \\
\hline Gender (female vs. male) & 0.661 & $0.379-1.153$ & 0.1448 \\
\hline NYHA functional class (III vs. II) & 1.805 & $1.057-3.086$ & 0.0306 \\
\hline Hypertension & 1.602 & $0.876-2.923$ & 0.1257 \\
\hline Diabetes mellitus & 0.935 & $0.531-1.648$ & 0.8167 \\
\hline Heart rate & 1.002 & $0.977-1.027$ & 0.8928 \\
\hline eGFR (Per 1-SD increase) & 0.709 & $0.523-0.944$ & 0.0157 \\
\hline BNP (Per 1-SD increase) & 1.339 & $1.089-1.532$ & 0.0010 \\
\hline H-FABP (Per 1-SD increase) & 1.441 & $1.228-1.691$ & $<0.0001$ \\
\hline Troponin T (Per 1-SD increase) & 1.078 & $0.894-1.299$ & 0.4304 \\
\hline LAD (Per 1-SD increase) & 1.361 & $0.823-2.238$ & 0.2317 \\
\hline LVEDD (Per 1-SD increase) & 1.150 & $0.895-1.480$ & 0.2816 \\
\hline LVEF (Per 1-SD increase) & 0.782 & $0.596-1.038$ & 0.0804 \\
\hline \multicolumn{4}{|l|}{ Multivariate analysis for $H-F A B P$} \\
\hline Age (Per 1-year increase) & 1.021 & $0.993-1.049$ & 0.1430 \\
\hline NYHA functional class (III vs. II) & 0.919 & $0.476-1.776$ & 0.8020 \\
\hline eGFR (Per 1-SD increase) & 0.926 & $0.679-2.494$ & 0.6736 \\
\hline H-FABP (Per 1-SD increase) & 1.278 & $1.035-1.584$ & 0.0231 \\
\hline BNP (Per 1-SD increase) & 1.133 & $0.426-1.406$ & 0.2934 \\
\hline \multicolumn{4}{|l|}{ Multivariate analysis for Troponin $T$} \\
\hline Age (Per 1-year increase) & 1.017 & $0.987-1.047$ & 0.2838 \\
\hline NYHA functional class (III vs. II) & 0.834 & $0.422-1.645$ & 0.6033 \\
\hline eGFR (Per 1-SD increase) & 0.858 & $0.604-1.209$ & 0.3832 \\
\hline Troponin T (Per 1-SD increase) & 1.045 & $0.859-1.271$ & 0.6562 \\
\hline BNP (Per 1-SD increase) & 1.246 & $1.000-1.532$ & 0.0407 \\
\hline
\end{tabular}

BNP: brain natriuretic peptide, eGFR: estimated glomerular filtration rate, H-FABP: heart-type fatty acid binding protein, LAD: left atrial dimension, LVEDD: left ventricular end-diastolic dimension, LVEF: left ventricular ejection fraction, NYHA: New York Heart Association, SD: standard deviation 
Table 4. The Optimal Cut-off Value to Predict Cardiovascular Events Determined by Receiver Operating Characteristics Curve Analysis

\begin{tabular}{llcccc}
\hline Subjects & Variables & Cut-off value & Sensitivity & Specificity & AUC \\
\hline CHF-AF & H-FABP & 5.4 & 63 & 73 & 0.73 \\
& Troponin T & 0.030 & 60 & 73 & 0.70 \\
CHF-SR & H-FABP & 4.6 & 69 & 72 & 0.75 \\
& Troponin T & 0.013 & 55 & 78 & 0.64 \\
\hline
\end{tabular}

AF: atrial fibrillation, AUC: area under the curve, CHF: chronic heart failure, H-FABP: heart-type fatty acid binding protein, SR: sinus rhythm

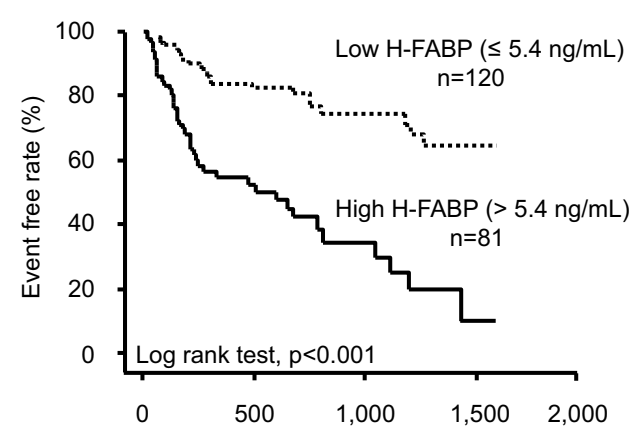

C

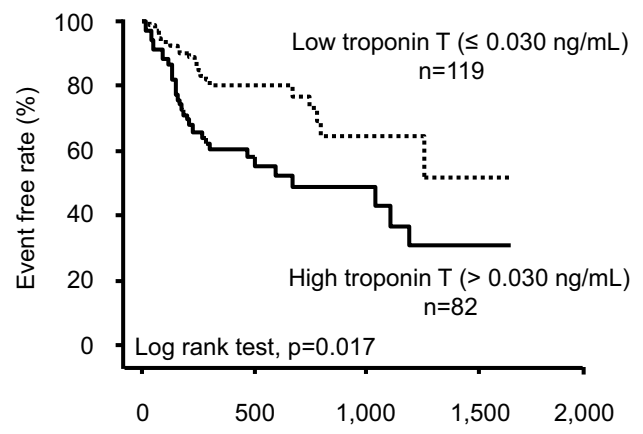

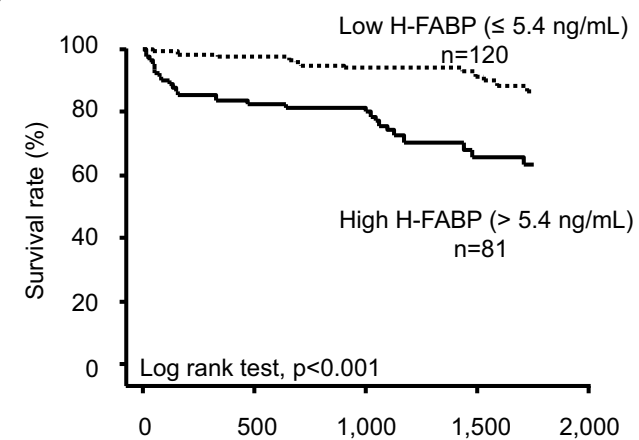

D

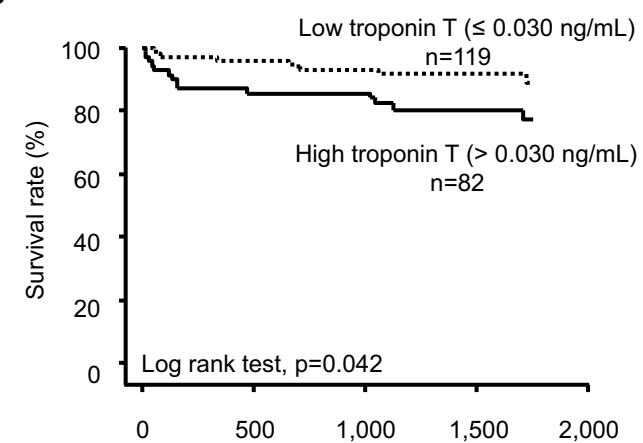

Figure 1. A Kaplan-Meier analysis of the time to subsequent cardiovascular events in the patients with CHF-AF. A: The cardiovascular event-free rate was greater in the patients with a low level of heart-type fatty acid binding protein (H-FABP) $(\leq 5.4 \mathrm{ng} / \mathrm{mL})$ than in those with a high level of $\mathrm{H}$ FABP $(>5.4 \mathrm{ng} / \mathrm{mL})$. B: The survival rate was greater in the patients with a low H-FABP level than in those with a high H-FABP level. $C$ : The cardiovascular event-free rate was greater in the patients with a low troponin $\mathrm{T}$ level $(\leq 0.030 \mathrm{ng} / \mathrm{mL})$ than in those with a high troponin $\mathrm{T}$ level $(>0.030 \mathrm{ng} / \mathrm{mL})$. D: The survival rate was greater in the patients with a low troponin $T$ level than in those with a high troponin T level.

the level of H-FABP, but not troponin T, was an independent predictor of cardiovascular events after adjusting for age, NYHA functional class, eGFR and BNP (H-FABP: hazard ratio, 1.278; 95\% confidence interval, 1.035-1.584; $\mathrm{p}=$ 0.0231) (Table 3).

\section{Comparison of the optimal cut-off values between the CHF-AF and CHF-SR patients}

As shown in Table 4, the ROC curve analyses revealed that a serum H-FABP level of $5.4 \mathrm{ng} / \mathrm{mL}$ and a serum troponin $\mathrm{T}$ level of $0.030 \mathrm{ng} / \mathrm{mL}$ were the threshold values for all cardiovascular events in the CHF-AF patients (H-FABP: sensitivity, $63 \%$; specificity, $73 \%$; area under the curve, 0.73 and troponin T: sensitivity, $60 \%$; specificity, $73 \%$; area un- der the curve, 0.70). Meanwhile, the ROC analyses revealed that a serum H-FABP level of $4.6 \mathrm{ng} / \mathrm{mL}$ and a serum troponin $\mathrm{T}$ level of $0.013 \mathrm{ng} / \mathrm{mL}$ were the threshold values for all cardiovascular events in the CHF-SR patients (H-FABP: sensitivity, 69\%; specificity, $72 \%$; area under the curve, 0.75 and troponin T: sensitivity, 55\%; specificity, 78\%; area under the curve, 0.64).

Among the patients with CHF-AF, a Kaplan-Meier analysis demonstrated that those with an elevated H-FABP level $(>5.4 \mathrm{ng} / \mathrm{mL})$ had a significantly higher rate of cardiovascular events and all-cause mortality compared to those without (Fig. 1A, B). Similarly, the patients with an elevated troponin $\mathrm{T}$ level $(>0.030 \mathrm{ng} / \mathrm{mL})$ also exhibited a higher rate of cardiovascular events and all-cause mortality than those 


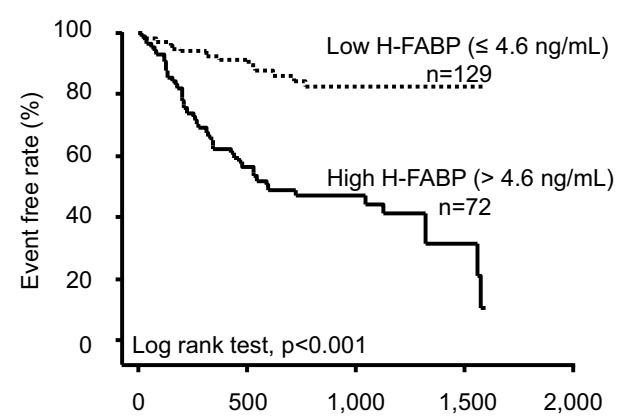

C

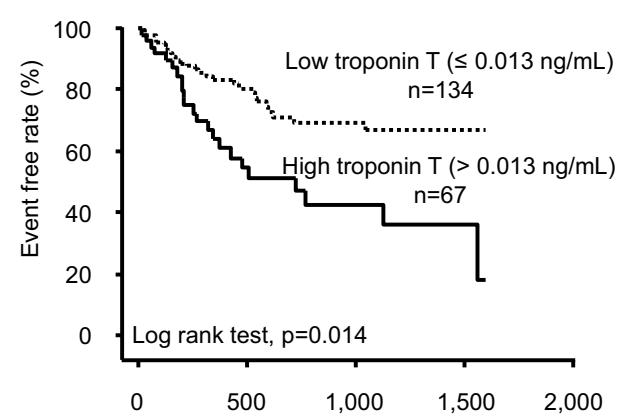

B

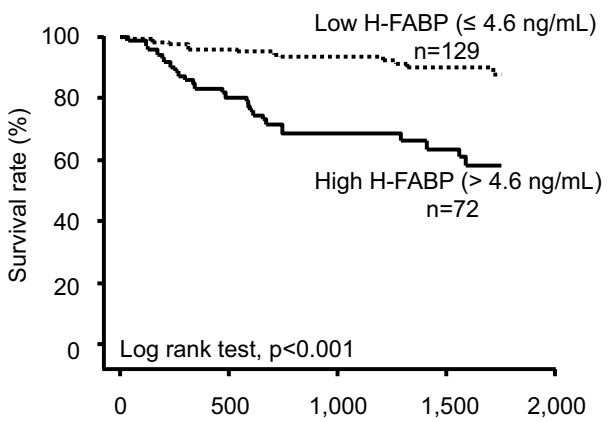

D

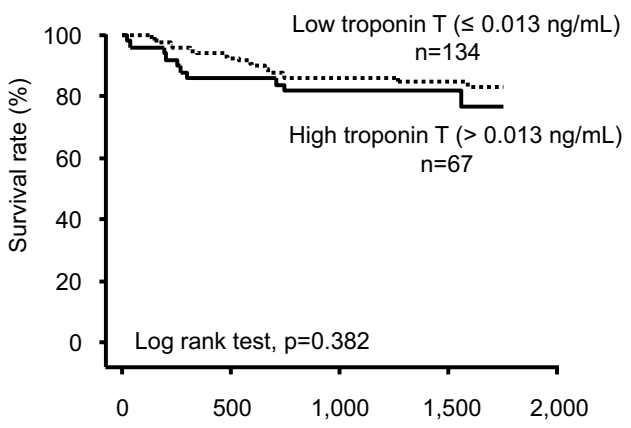

Figure 2. A Kaplan-Meier analysis of the time to subsequent cardiovascular events in the patients with CHF-SR. A: The cardiovascular event-free rate was greater in the patients with a low level of heart-type fatty acid binding protein $(\mathrm{H}-\mathrm{FABP})(\leq 4.6 \mathrm{ng} / \mathrm{mL})$ than in those with a high level of $\mathrm{H}$ FABP ( $>4.6 \mathrm{ng} / \mathrm{mL})$. B: The survival rate was greater in the patients with a low H-FABP level than in those with a high H-FABP level. $\mathrm{C}$ : The cardiovascular event-free rate was greater in the patients with a low troponin $\mathrm{T}$ level $(\leq 0.013 \mathrm{ng} / \mathrm{mL})$ than in those with a high troponin $\mathrm{T}$ level $(>0.013 \mathrm{ng} / \mathrm{mL})$. D: There were no significant differences in the survival rate between the patients with a low troponin $T$ level and those with a high troponin $T$ level.

without (Fig. 1C, D). Among the patients with CHF-SR, a Kaplan-Meier analysis demonstrated that those with an elevated H-FABP level $(>4.6 \mathrm{ng} / \mathrm{mL})$ had a significantly higher rate of cardiovascular events and all-cause mortality compared to those without (Fig. 2A, B). Similarly, the patients with an elevated troponin $\mathrm{T}$ level $(>0.013 \mathrm{ng} / \mathrm{mL})$ also exhibited a higher rate of cardiovascular events than those without (Fig. 2C). On the other hand, there were no significant differences in all-cause mortality between the patients with an elevated troponin $\mathrm{T}$ level and those without (Fig. 2D).

\section{Discussion}

\section{Main findings}

The findings of this study were as follows: 1) the CHFAF patients had higher H-FABP and troponin $\mathrm{T}$ levels than those with CHF-SR, despite their similar baseline characteristics; 2) the H-FABP and troponin T levels were independent predictors of cardiovascular events in both the CHF-AF and CHF-SR patients; and 3) the optimal cut-off values for the levels of H-FABP and troponin $\mathrm{T}$ for predicting cardiovascular events were higher in the CHF-AF patients than in the CHF-SR patients.

\section{Mechanisms of myocardial damage in AF patients}

Ventricular cell loss has been confirmed in AF patients with tachycardia-induced cardiomyopathy $(15,16)$. However, in the present study, myocardial injury was confirmed in the CHF-AF patients, irrespective of whether the heart rate was strictly controlled. Therefore, other mechanisms should be considered to explain the relationship between myocardial damage and AF. Cardiac sympathetic nervous activation is associated with myocardial injury (17). Furthermore, AF-induced sympathetic nervous disintegrity is one process underlying the progression of myocardial damage. Cardiac sympathetic nervous scintigraphy findings show that AF directly activates the cardiac sympathetic nervous function in patients with lone AF (18). AF-induced apoptosis in cardiomyocytes may also be a cause of myocardial injury. In addition, it has previously been reported that cardiac cell apoptosis occurs in AF model animals. Interestingly, a previous report showed that markers of serum apoptosis, including the levels of apoptosis-stimulating fragment and tumor necrosis-related apoptosis-inducing ligand, are decreased following successful AF ablation (19). Ongoing myocardial damage has been recognized to be an important process in 
the progression of $\mathrm{CHF}(20,21)$. Therefore, AF-induced myocardial damage may play a pivotal role in $\mathrm{CHF}$ progression.

\section{Clinical outcomes and myocardial damage in CHF- AF patients}

Despite the fact that no differences were found in the baseline characteristics between the CHF-AF and CHF-SR patients in this study, the biomarkers of myocardial damage were significantly higher in the CHF-AF patients than in the CHF-SR patients. In addition, the cut-off values for the levels of H-FABP and troponin $\mathrm{T}$ were higher in the CHF-AF patients than in the CHF-SR patients. These findings suggest that the presence of $\mathrm{AF}$ is an important cause of myocardial damage. Although the prognosis is poor in CHF-AF patients, no clinical trials have investigated prognostic markers of cardiovascular events in this population. Previous studies have reported that markers of myocardial damage are associated with cardiovascular events in patients with a broad spectrum of heart diseases $(20,22-25)$. The results of the present study also demonstrate that both the H-FABP and troponin $\mathrm{T}$ levels can be used to risk stratify CHF-AF patients.

The H-FABP level had a larger AUC than the troponin $\mathrm{T}$ level among the CHF-AF and CHF-SR patients. Basic and clinical research using rats and human autopsy cases has found that leakage of H-FABP occurs despite the absence of myocyte necrosis (26). H-FABP is a low molecular weight protein that is confined to the cytoplasm and is released into the circulation through the porous membranes of damaged myocardial cells (22). The presence of AF is associated with slight elevation of the levels of myocardial damage markers. Therefore, monitoring the circulating H-FABP levels may be a more useful strategy than monitoring the troponin $\mathrm{T}$ levels for identifying high-risk patients with CHF-AF.

\section{Study limitations}

First, we were unable to confirm the precise duration of AF. Patients with long-standing AF may have relatively elevated serum H-FABP levels compared to those with shortterm AF. Second, the serum H-FABP level was measured once in each patient when the patient was stable. Obtaining additional H-FABP measurements during the follow-up period may be useful. Third, although the heart rates on the day of blood sampling were similar between the CHF-AF and CHF-SR patients, we did not confirm the heart rates during exercise. Fourth, we did not examine Holter recordings or the total, maximum, minimum and average heart rates in the present study. Finally, further studies of these populations are required to determine whether aggressive rhythm control therapy reduces the incidence of myocardial damage, myocardial remodeling and subsequent cardiovascular events.

\section{Conclusion}

Myocardial damage, as indicated by the serum H-FABP and troponin $\mathrm{T}$ levels, is deteriorated by the presence of $\mathrm{AF}$. Myocardial damage markers are independent predictors of future cardiovascular events in patients with CHF-AF.

The authors state that they have no Conflict of Interest (COI).

\section{References}

1. Olsson LG, Swedberg K, Ducharme A, et al. Atrial fibrillation and risk of clinical events in chronic heart failure with and without left ventricular systolic dysfunction: results from the Candesartan in Heart failure-Assessment of Reduction in Mortality and morbidity (CHARM) program. J Am Coll Cardiol 47: 1997-2004, 2006.

2. Zakeri R, Chamberlain AM, Roger VL, et al. Temporal relationship and prognostic significance of atrial fibrillation in heart failure patients with preserved ejection fraction: a community-based study. Circulation 128: 1085-1093, 2013.

3. Schotten U, Ausma J, Stellbrink C, et al. Cellular mechanisms of depressed atrial contractility in patients with chronic atrial fibrillation. Circulation 103: 691-698, 2001.

4. Krahn AD, Manfreda J, Tate RB, Mathewson FA, Cuddy TE. The natural history of atrial fibrillation: incidence, risk factors, and prognosis in the Manitoba Follow-Up Study. Am J Med 98: 476484, 1995.

5. Tsang TS, Gersh BJ, Appleton CP, et al. Left ventricular diastolic dysfunction as a predictor of the first diagnosed nonvalvular atrial fibrillation in 840 elderly men and women. J Am Coll Cardiol 40: 1636-1644, 2002.

6. Sanders P, Morton JB, Davidson NC, et al. Electrical remodeling of the atria in congestive heart failure: electrophysiological and electroanatomic mapping in humans. Circulation 108: 1461-1468, 2003.

7. Cha YM, Redfield MM, Shen WK, Gersh BJ. Atrial fibrillation and ventricular dysfunction: a vicious electromechanical cycle. Circulation 109: 2839-2843, 2004.

8. Kostin S, Pool L, Elsasser A, et al. Myocytes die by multiple mechanisms in failing human hearts. Circ Res 92: 715-724, 2003.

9. Olivetti G, Abbi R, Quaini F, et al. Apoptosis in the failing human heart. N Engl J Med 336: 1131-1141, 1997.

10. Fuster V, Ryden LE, Cannom DS, et al. 2011 ACCF/AHA/HRS focused updates incorporated into the ACC/AHA/ESC 2006 guidelines for the management of patients with atrial fibrillation: a report of the American College of Cardiology Foundation/American Heart Association Task Force on practice guidelines. Circulation 123: e269-e367, 2011.

11. Niizeki $T$, Takeishi $Y$, Arimoto $T$, et al. Heart-type fatty acidbinding protein is more sensitive than troponin $\mathrm{T}$ to detect the ongoing myocardial damage in chronic heart failure patients. J Card Fail 13: 120-127, 2007.

12. Arimoto T, Takeishi $Y$, Niizeki T, et al. Cardiac sympathetic denervation and ongoing myocardial damage for prognosis in early stages of heart failure. J Card Fail 13: 34-41, 2007.

13. Giannitsis E, Kurz K, Hallermayer K, Jarausch J, Jaffe AS, Katus HA. Analytical validation of a high-sensitivity cardiac troponin $T$ assay. Clin Chem 56: 254-261, 2010.

14. Kawai M, Yoshimura M, Harada M, et al. Determination of the Btype natriuretic peptide level as a criterion for abnormalities in Japanese individuals in routine clinical practice: the J-ABS MultiCenter Study (Japan Abnormal BNP Standard). Intern Med 52: 171-177, 2013. 
15. Trappe K, Thomas D, Bikou O, et al. Suppression of persistent atrial fibrillation by genetic knockdown of caspase 3: a preclinical pilot study. Eur Heart J 34: 147-157, 2013.

16. Bauer A, McDonald AD, Donahue JK. Pathophysiological findings in a model of persistent atrial fibrillation and severe congestive heart failure. Cardiovasc Res 61: 764-770, 2004.

17. Chiu YT, Chen YT, Lin NN, et al. Sympathetic activity and myocardial damage after stimulation of dorsal medulla and vagotomy in a novel animal model. Int J Cardiol 100: 401-407, 2005.

18. Arimoto $\mathrm{T}$, Tada $\mathrm{H}$, Igarashi $\mathrm{M}$, et al. High washout rate of iodine-123-metaiodobenzylguanidine imaging predicts the outcome of catheter ablation of atrial fibrillation. J Cardiovasc Electrophysiol 22: 1297-1304, 2011.

19. Osmancik P, Peroutka Z, Budera P, et al. Decreased apoptosis following successful ablation of atrial fibrillation. Cardiology 116: 302-307, 2010.

20. Arimoto T, Takeishi Y, Shiga R, et al. Prognostic value of elevated circulating heart-type fatty acid binding protein in patients with congestive heart failure. J Card Fail 11: 56-60, 2005.

21. Kusumoto A, Miyata M, Kubozono T, et al. Highly sensitive car- diac troponin $\mathrm{T}$ in heart failure: comparison with echocardiographic parameters and natriuretic peptides. J Cardiol 59: 202208, 2012.

22. Viswanathan K, Kilcullen N, Morrell C, et al. Heart-type fatty acid-binding protein predicts long-term mortality and re-infarction in consecutive patients with suspected acute coronary syndrome who are troponin-negative. J Am Coll Cardiol 55: 2590-2598, 2010.

23. Kutsuzawa $\mathrm{D}$, Arimoto $\mathrm{T}$, Watanabe $\mathrm{T}$, et al. Ongoing myocardial damage in patients with heart failure and preserved ejection fraction. J Cardiol 60: 454-461, 2012.

24. Daidoji $\mathrm{H}$, Arimoto T, Nitobe J, et al. Circulating heart-type fatty acid binding protein levels predict the occurrence of appropriate shocks and cardiac death in patients with implantable cardioverterdefibrillators. J Card Fail 18: 556-563, 2012.

25. Sato Y, Fujiwara H, Takatsu Y. Cardiac troponin and heart failure in the era of high-sensitivity assays. J Cardiol 60: 160-167, 2012.

26. Meng X, Ming M, Wang E. Heart fatty acid binding protein as a marker for postmortem detection of early myocardial damage. Forensic Sci Int 160: 11-16, 2006.

(C) 2014 The Japanese Society of Internal Medicine http://www.naika.or.jp/imonline/index.html 